\title{
The role of electrohydrodynamic forces in the dielectrophoretic manipulation and separation of particles
}

\author{
A. Ramos ${ }^{+}$, H. Morgan*, N.G. Green* and A. Castellanos ${ }^{+}$ \\ *Bioelectronics Research Centre, \\ Department of Electronics and Electrical Engineering, Rankine Building, Oakfield Avenue, \\ University of Glasgow, Glasgow G12 8LT, UK \\ ${ }^{+}$Departamento de Electronica y Electromagnetismo \\ Universidad de Sevilla, Avda. Reina Mercedes, s/n 41012-Sevilla, SPAIN
}

\begin{abstract}
A dielectric particle suspended in a dielectric medium polarises under the influence of an electric field. If the field is non-uniform, the particle experiences a directional force and the resulting movement is referred to as dielectrophoresis. The high electric fields can also cause fluid motion, which in turn results in a viscous drag force on the particle. This paper outlines the application of dielectrophoresis to the sub-micrometre scale and discusses mechanisms responsible for fluid motion in small electrode structures. Preliminary measurements of fluid velocity in a microelectrode array are presented and are discussed in relation to theoretical models. Finally the relevance of electric field induced fluid motion to particle separation technologies is discussed.
\end{abstract}

\section{INTRODUCTION}

When a polarisable particle, suspended in an electrolyte, is subjected to an electric field, a dipole moment is induced on the particle. For a spatially non-uniform AC electric field, the interaction of the dipole moment and the electric field gradient causes movement of the particle, termed dielectrophoresis (DEP) by Pohl [1]. Since the early pioneering work of Pohl, DEP technology has advanced rapidly, primarily due to the advent of methods for the fabrication of miniaturised microelectrode systems. Such systems are capable of providing high electric fields with small applied voltages. It has been shown that a wide range of particles such as cells, latex spheres, and microorganisms can be selectively manipulated using dielectrophoretic techniques [1-3] and more recently, sub-micrometre particles such as latex spheres, macromolecules, DNA and viruses have been manipulated and characterised [4-10]. For sub-micrometre particles it was believed Brownian motion would prevent deterministic 
movement by DEP, but this premise has been disproved. However, substantial electric field gradients are required to produce sufficient force to move the particles [10], and these high fields have the unavoidable side effect of causing electrically driven fluid movement which can also cause particle movement. Such fluid-driven particle movement has been observed and reported previously $[11,12]$. Recently the flow has been exploited, in combination with DEP forces, to provide a new method for separating particles on microelectrode arrays [11]. Experimental images of the flow patterns have been published [12], together with the postulation that the flow could be related to an $\mathrm{AC}$ electroosmotic process. However, the frequency dependent behaviour of the fluid flow was not measured.

In this paper, experimental measurements of fluid driven particle movement are reported for the first time, together with measurements of the frequency-dependent impedance of microelectrodes. A brief summary of the theory of dielectrophoresis and two mechanisms responsible for fluid flow (electrothermal and electroosmotic forces) are presented. A simple explanation for $\mathrm{AC}$ electroosmotic driven particle movement is outlined and compared with new experimental data as well as previously reported observations $[11,12]$.

\section{EXPERIMENTAL METHOD}

The electrode used in the experiments comprised two parallel fingers, $2 \mathrm{~mm}$ long, $100 \mu \mathrm{m}$ wide, separated by a gap of $25 \mu \mathrm{m}$. The electrodes were fabricated on glass substrates using photolithography and consisted of a $10 \mathrm{~nm}$ layer of titanium and a $100 \mathrm{~nm}$ layer of gold. The electric field was generated using a variable frequency/amplitude signal generator. The suspending electrolyte consisted of water with varying amounts of $\mathrm{KCl}$ added to control the conductivity. For the experiments, two different solutions of $\mathrm{KCl}$ were used with conductivities of $2.2 \mathrm{mSm}^{-1}$ and $7.8 \mathrm{mSm}^{-1}$. In electrolytic solutions, below approximately $1 \mathrm{kHz}$ and at high potentials, electrochemical dissolution of the gold electrodes occurs. To prevent this, electrodes were also fabricated covered by an insulating layer of silicon nitride (deposited using a low temperature plasma process) to a thickness of approximately $70 \mathrm{~nm}$. A HewlettPackard 4192A impedance analyser was used to measure the impedance of the electrode arrays at frequencies up to $10 \mathrm{MHz}$.

Fluid flow patterns were observed using fluorescently loaded $282 \mathrm{~nm}$ diameter latex particles (Molecular Probes). First, qualitative observations of the behaviour of the fluid and the particles as a function of applied signal strength and frequency were made over a range of values. Second, keeping the applied signal strength constant at 10 volts peak to peak, the position of individual $282 \mathrm{~nm}$ diameter latex particles was recorded as a function of time and applied signal frequency using a fluorescence microscope and video camera. In order to quantify the particle velocity, an arbitrary point $10 \mu \mathrm{m}$ in from the edge of the electrode was chosen. Only particles moving in the focal plane of the microscope objective were recorded, giving heights for the particles of up to approximately $1 \mu \mathrm{m}$. The velocity was calculated for several particles at each frequency and the average value taken. 


\section{RESULTS}

For both electrolyte conductivities, applied potentials up to 10 Volts peak to peak and for frequencies greater than $\sim 3 \mathrm{MHz}$, it was observed that particles moved away from regions of high electric field gradient under the influence of negative DEP forces [8]. In addition, for an applied signal of 10 volts peak to peak, small fluid flow patterns were observed across the electrode edges. For frequencies below $1 \mathrm{MHz}$ and low potentials, the particles were trapped under positive DEP forces at the electrode edges. For higher potentials, the particles moved from the inter-electrode gap across the electrode and onto the electrode surface as shown schematically in figure 1 .

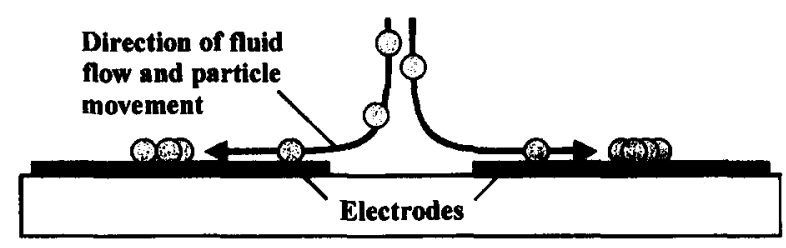

Figure 1. Diagram illustrating the motion of particles induced by fluid flow for frequencies below $1 \mathrm{MHz}$. The particles move down from the bulk into the inter-electrode gap and out onto the electrode surface, with a velocity that varies with frequency and position.

The direction of fluid flow was always constant, but the magnitude was seen to depend on frequency and the applied voltage. The magnitude of the fluid velocity was maximum at a certain frequency (see figure 2) and tended to zero at high and low frequency limits. For the insulated electrodes, particle velocities were observed to peak at a higher frequency of approximately $100 \mathrm{kHz}$.

Measurements of particle velocities were made using the bare gold electrodes for both electrolyte conductivities and the data is summarised in figure 2 . It can be seen that over the frequency range $1 \mathrm{kHz}$ to $1 \mathrm{MHz}$, particle velocities increased with decreasing frequency, reaching a maximum at a frequency that changed with the medium conductivity. The maximum velocity observed was of the order of $100 \mu \mathrm{m} / \mathrm{s}$.

The fluid flow causes particles to move away from the electrode edges as illustrated in figure 1. A photograph showing this effect can be seen in figure 3 which shows an experimental image of $557 \mathrm{~nm}$ diameter latex spheres on castellated electrodes $[9,11]$ with the particles lying in patterns on top of the electrodes. In this image the frequency of the applied signal was $50 \mathrm{kHz}$ and the potential 5 volts. On this shape of electrode, commonly used for dielectrophoretic separation, the flow patterns push the particles into regular formations on the electrode surface. Where the electrodes are symmetrical, the particles form diamond patterns, an effect similar to that observed for larger particles on larger electrodes [17] but which has remained unexplained. We ascribe this effect to electric field induced fluid flow. 


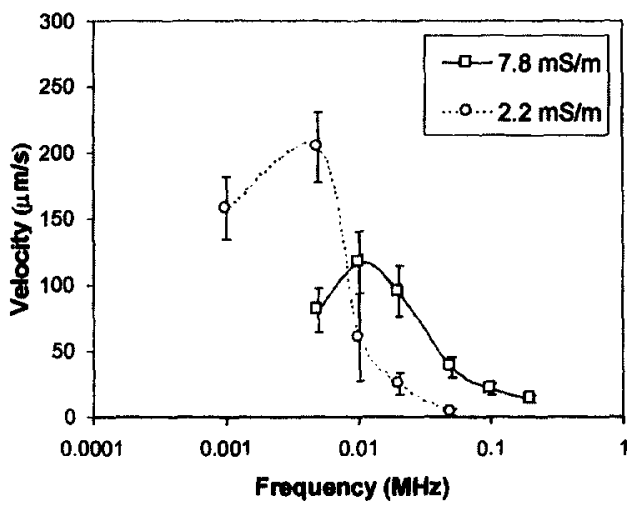

Figure 2. Plot of the velocity of $282 \mathrm{~nm}$ latex spheres as a function of frequency measured at a point $10 \mu \mathrm{m}$ from the edge of a $25 \mu \mathrm{m}$ gap finger electrode. The voltage was 10 Volts peak to peak.

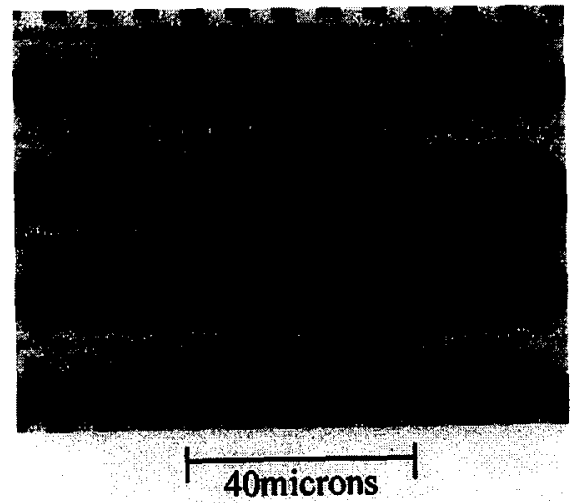

Figure 3. Experimental image of $557 \mathrm{~nm}$ diameter latex spheres experiencing fluid flow on castellated electrodes and forming diamond patterns on symmetrical electrodes.

\section{DISCUSSION}

For the parallel wire electrode geometry used in this work, the measured fluid velocities at frequencies greater than $0.5 \mathrm{MHz}$ were typically $1 \mu \mathrm{m} / \mathrm{sec}$ or less (with 10 volts peak to peak and a medium conductivity $=7.8 \mathrm{mSm}^{-1}$ ) and generally invariant with frequency. This velocity is of the same order of magnitude as that produced by the DEP force acting on a $282 \mathrm{~nm}$ diameter particle at a point $10 \mu \mathrm{m}$ away from the electrode edge (see Appendix equation (A1) and [12]). It is probable that these fluid movements are induced by electrothermal effects. Closer to the electrode edge the dielectrophoretic force increases rapidly (almost exponentially), so that in most cases the effect of electrothermal forces on particles such as the spheres used in this work can be neglected. For much smaller particles and/or higher fields this may not always be the case.

The magnitude of fluid flow at low frequencies (summarised in figure 2) is much greater than the equivalent DEP velocity of a $282 \mathrm{~nm}$ particle. Also preliminary measurements indicate that the frequency and conductivity dependence of the fluid flow is inconsistent with that expected from electrothermal effects [12]. For electrothermally induced fluid flow and for frequencies below and above $f_{c}$ (see Appendix, equation (A3)), the fluid velocity should be independent of frequency.

The low-frequency motion of the fluid can be explained through the effect of a mechanisms analogous to electroosmosis. In non-uniform electric fields, a component of the field will lie parallel to the electrode surface (i.e. there exists a tangential electric field). This field will interact with the electrical double layer on the electrodes to produce a force. The principle of this effect, which is referred to here as AC electroosmosis, is illustrated in figure 4. It can be seen from this figure that the direction of the force is always away from the regions of high tangential field and across the electrode surface. Since the direction of the force vector 
remains constant regardless of the sign of the electric field, it can therefore be inferred that the direction of fluid flow will remain constant in an $\mathrm{AC}$ field.

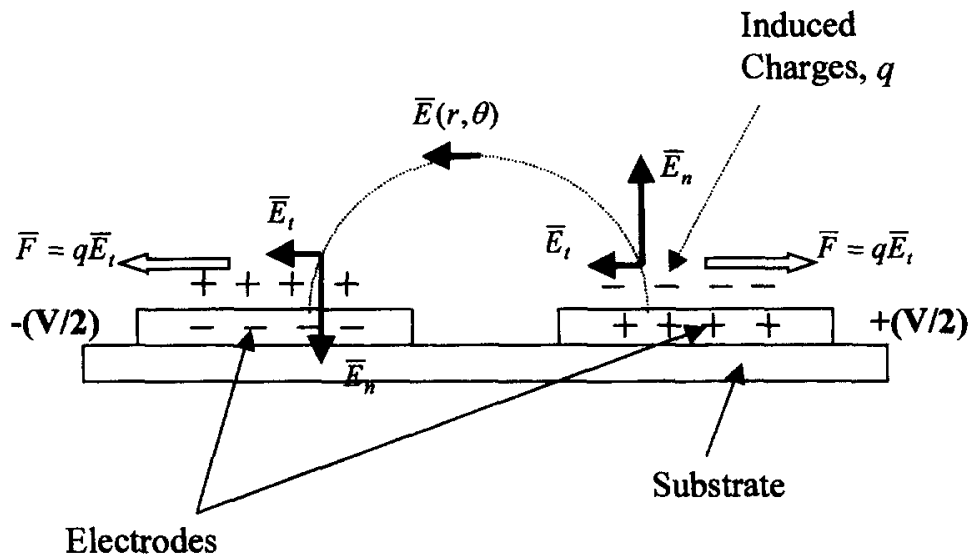

Figure 4. Diagrammatic representation of the movement of charge in the double layer due to the action of a tangential electric field.

Owing to the divergent field geometry, the charge density on the electrode will not be constant but will vary with the magnitude of the electric field, being highest at the electrode edge. Also, due to double layer polarisation effects $[13,14]$ both $E_{\mathrm{t}}$ and $\sigma_{\mathrm{q}}$ will vary with the applied frequency. For electroosmosis in DC fields, in the linear approximation, the velocity of a fluid of viscosity $\eta$ is given by:

$v=\frac{E_{t} \sigma_{q}}{k \eta}$

where $E_{\mathrm{t}}$ is the tangential electric field at the Debye length $\boldsymbol{\kappa}^{-1}$, and $\sigma_{\mathrm{q}}$ is the equivalent surface charge density in the diffuse double layer. In $\mathrm{AC}$ fields, the velocity will be given by:

$v=\frac{1}{2 \kappa \eta} \operatorname{Re}\left[\sigma_{q} E_{t}^{*}\right]$

where $E_{t}^{*}$ is the complex conjugate of the tangential electric field phasor. This equation can be used to give a first order approximation for the frequency and field dependence of the $\mathrm{AC}$ electroosmotic velocity.

The frequency dependence of the fluid flow can be explained by considering the variation of the double layer potential and charge density with frequency. Again using a linear approximation, the double layer charge density is given by $\sigma_{q}=\varepsilon \kappa V_{d}$ where $V_{d}$ is the potential across the double layer. In order to estimate the double layer voltage, the double layer/medium can be analysed as a series combination of two impedances: the double layer $Z_{d}$ 
and the medium $Z_{m}$ [15]. To a first approximation the double layer can be considered to be a capacitor with reactive impedance $Z_{d}=1 / j \omega C_{d}$ where $C_{d}=\varepsilon \times \mathrm{S}$ (S is the surface area). The medium is taken to be an impedance consisting of a resistance, $R_{m}$ and a capacitance $C_{m}$ in parallel.

The frequency-dependent impedance of the electrode array in $7.8 \mathrm{mSm}^{-1} \mathrm{KCl}$ was measured. At high frequencies the double layer impedance becomes much smaller than the medium impedance and $R_{m}$ and $C_{m}$ were obtained. At frequencies below the charge relaxation frequency i.e. $\omega \varepsilon / \sigma<<1, Z_{m}$ approximates to $R_{m}$ with a good degree of accuracy. Using this value for $Z_{m}$, the frequency-dependent voltage across the double layer and the medium was then calculated. The real and imaginary parts of the normalised voltage across the medium are shown in figure 5 , curve (a).

It can be seen from this figure that at high frequencies $\left(\omega C_{\mathrm{d}} R_{\mathrm{m}} \gg 1\right)$, the applied voltage is dropped entirely across the medium, while at low frequencies $\left(\omega C_{\mathrm{d}} R_{\mathrm{m}}<1\right)$ the voltage is dropped across the double layer. At high frequencies the charge in the double layer tends to zero and according to equation (2) the AC electroosmotic flow will also tend to zero, in agreement with the experimental data (see figure 2). At low frequencies the electric field in the medium tends to zero. Since the tangential electric field across the double layer-medium interface is continuous, the tangential component of the electric field in the double layer will also tend to zero at low frequencies and again electroosmotic flow will cease. Measurements indicate that the fluid velocity reaches a maximum value but there is insufficient data to demonstrate the low frequency behaviour.

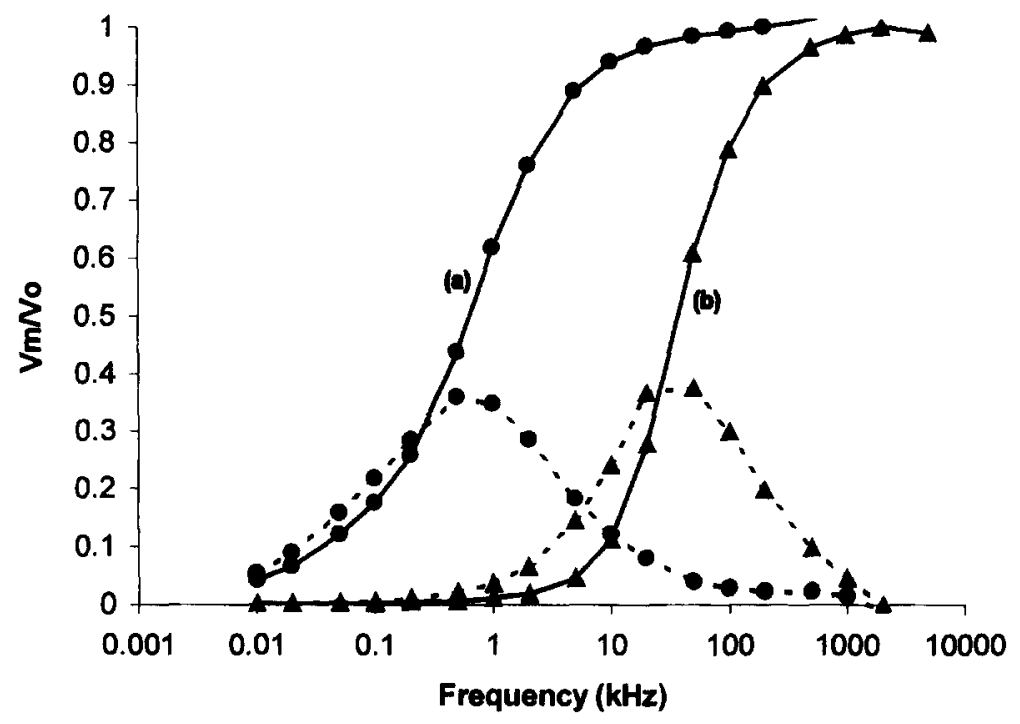

Figure 5. The real and imaginary components of the normalised voltage in the medium as a function of frequency for the gold interdigitated electrodes (a) and the silicon nitride insulated electrodes (b). In each case, the real component is given by the solid line and the imaginary component by the dashed line. The medium conductivity was $7.8 \mathrm{mSm}^{-1}$. 
Over the range of frequencies around $\omega=1 /\left(C_{\mathrm{d}} R_{\mathrm{m}}\right)$, there is both a significant charge in the double layer and electric field in the medium. Therefore, sufficient tangential electric field acts on the double layer to result in charge movement and fluid flow. The data plotted in figure 5(a) for the gold interdigitated electrode shows that the characteristic frequency of the system:

$f=\frac{1}{2 \pi C_{d} R_{m}}$

is of the order of $1 \mathrm{kHz}$.

From figure 2 it can also be seen that the frequency of maximum fluid velocity increases as the conductivity increases. Equation (3) indicates that the characteristic frequency varies with the square root of the conductivity $\left(\mathrm{R}_{\mathrm{m}} \propto \sigma\right.$ and $C_{d} \propto 1 / \sqrt{ } \sigma$ ). This provides further evidence in support of the existence of $\mathrm{AC}$ electroosmosis.

Also shown in figure 5, curve (b) are the real and imaginary components of the voltage across the medium for the insulated electrodes. These are again calculated from measurements of the impedance of the system. In this case the double layer impedance is now in series with the silicon nitride layer which acts as an additional impedance, both of which are assumed to be purely capacitive. The capacitance of the layer above the electrodes is therefore equal to the series sum of the two capacitances. However, the capacitance of the silicon nitride layer $C_{\mathrm{n}}$ is much smaller than $C_{\mathrm{d}}$ since $C_{\mathrm{r}} / C_{\mathrm{d}}=\left(\varepsilon^{\prime} \varepsilon_{\mathrm{n}}\right) d \kappa \approx 125$, where $d$ is the thickness of the silicon nitride layer and $\varepsilon$ and $\varepsilon_{n}$ are the permittivities of water and silicon nitride respectively. The ratio between the characteristic frequencies for the insulating and normal electrodes (from equation (3)) can therefore be taken to be the ratio of the capacitances of the silicon nitride layer and the double layer i.e. 125. This is approximately the ratio between the measured characteristic frequencies as can be seen in figure 5 . This shift is also confirmed by observations of the movement of latex particles on silicon nitride electrodes where the frequency of maximum velocity was approximately $100 \mathrm{kHz}$.

\subsection{Fluid flow in a microseparation system.}

The dielectrophoretic separation of two particles can be accomplished by choosing an appropriate conductivity and frequency so that one particle experiences positive DEP and the other experiences negative DEP, the two particles moving in opposite directions. Providing fluid movement can be minimised, it is possible to manipulate particles of any size. If dielectrophoretic separation is performed at the frequency predicted by equation (A3) then electrothermally induced fluid flow could be eliminated (see Appendix and [12]). In addition, this frequency is much greater than the characteristic frequency of the medium/double layer so that AC electroosmotic effects are also insignificant. To illustrate this point the ClausiusMossotti factor (see Appendix), was calculated for two latex particles of different conductivities, $(0.1 \mathrm{~S} / \mathrm{m}$ and $0.01 \mathrm{~S} / \mathrm{m})$ and identical permittivities $\left(\varepsilon_{\mathrm{p}}=2.5\right)$. A plot of the frequency variation of the "degree of separation" between the two spheres is shown in figure 6a. In this plot, if both spheres experience either positive or negative DEP then the value is set to zero. Otherwise the magnitude of: $\operatorname{Re}\left\{\left[K_{\mathrm{A}}(\omega)-K_{\mathrm{B}}(\omega)\right]\right\}$ is plotted. It can be seen that there is an area in the conductivity/frequency plane in which the spheres would move in opposite directions. 


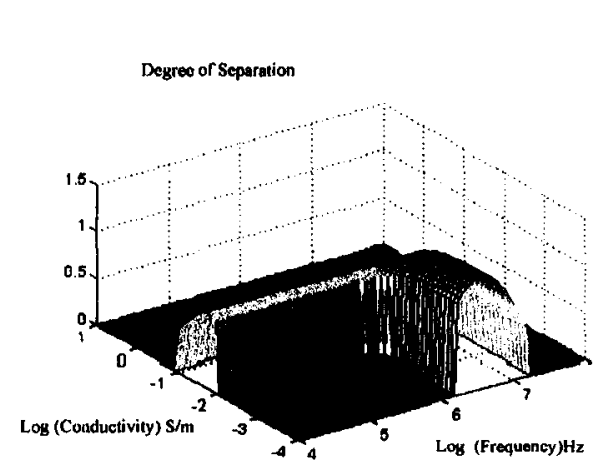

Figure 6a. A three dimensional plot showing how the "degree of separation" for two particles of different conductivity varies with frequency and medium conductivity.
Degree of Separation

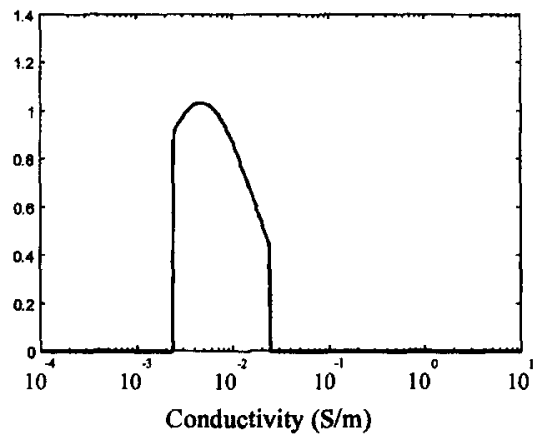

Figure 6b. The "degree of separation" of two particles shown in figure $6 \mathrm{a}$, plotted against medium conductivity at a frequency calculated for the zero EHD force given by equation (A3).

The plot in figure 6b shows the "degree of separation" plotted along a line given by the frequency of equation (A3). It can be seen that in the conductivity range of $2.5 \mathrm{mSm}^{-1}$ to $25 \mathrm{mSm}^{-1}$ the EHD force is zero and the particles can be separated.

\section{CONCLUSIONS}

The dielectrophoretic manipulation of sub-micron particles can be achieved by generating high strength electric fields, but this gives rise to fluid motion. The electrothermal fluid motion is small and can be considered insignificant.

It has been proposed that the fluid flow observed at low frequencies (below $0.5 \mathrm{MHz}$ ) is due to the action of a tangential field on the double layer, a mechanism that has been termed $\mathrm{AC}$ electroosmosis. Measurements have been performed using coplanar plate electrodes to characterise this flow for the first time. Circuit analysis and measurement of the impedance of the electrodes have been used to determine the voltage across the double layer as a function of frequency. It has been shown that there is a correlation between the flow velocity and the voltage across the medium and the charge in the double layer, both of which are frequency dependent. Further work is being carried out to completely characterise the fluid behaviour down to very low frequencies.

Fluid flow can harnessed for particle separation systems or eliminated by choosing appropriate frequency and conductivity regimes. Based on parameters described here, new separation systems for sub-micron particles will be developed. 


\section{Acknowledgements}

The authors wish to acknowledge financial support from the British Council and Acciones Integradas programme. We also wish to acknowledge the UK Biotechnology and Biological Sciences Research Council (grant no. 17/T05315) and the Spanish Dirección General de Enseffanza Superior (research project no. PB96-1375).

\section{APPENDIX - Electrical forces on particles and fluid}

For the convenience of the reader, a brief summary of the electrical forces on a particle and the fluid is included here. A more complete description can be found in reference [12].

\section{A1. Dielectrophoresis}

Consider a homogeneous spherical dielectric particle of radius $a$ suspended in an aqueous medium subjected to a harmonic electric field of single frequency $\omega$ The time averaged dielectrophoretic force on the particle is given by $[3,12]$ :

$\mathbf{F}_{D E P}(\omega)=2 \pi \varepsilon_{m} a^{3} \operatorname{Re}\left\{\frac{\widetilde{\varepsilon}_{p}-\widetilde{\varepsilon}_{m}}{\widetilde{\varepsilon}_{p}+2 \widetilde{\varepsilon}_{m}}\right\} \nabla\left|\mathbf{E}_{m s}\right|^{2}$

where $\mathbf{E}_{r m s}$ is the root mean square electric field, $\varepsilon_{m}$ is the real permittivity of the medium and $\widetilde{\varepsilon}_{p}$ and $\widetilde{\varepsilon}_{m}$ are the complex permittivities $(\widetilde{\varepsilon}=\varepsilon-j \sigma / \omega)$ of the particle and the medium respectively. The magnitude of the force depends on both $\nabla\left|\mathbf{E}_{m s}\right|^{2}$ and the real part of the term in curly brackets. The latter is referred to as the Clausius-Mossotti factor and varies with the frequency of the applied field bounded by -0.5 and +1 (for the particular case of a sphere). Positive DEP occurs when $\operatorname{Re}\{K(\omega)\}>0$; the force is towards increasing electric field strength and particles collect at electrode edges. The converse of this is negative DEP which occurs when $\operatorname{Re}\{K(\omega)\}<0$; the force is in the direction of decreasing field strength and the particles are repelled from the electrode edge.

\section{A2. Electrothermal forces}

In a conducting medium, the high electric fields required for dielectrophoretic manipulation of particles generate heat. The resulting temperature field will be inhomogeneous, and spatial variations in the density, electrical conductivity and permittivity will occur, leading to volume forces in the fluid. Gradients in conductivity and permittivity give rise to electrothermal forces, a complete description of which can be found in reference [12]. 
For a small parallel wire electrode, the fluid velocity is [12]:

$v \propto\left(\frac{\varepsilon \sigma}{\eta k T} \frac{V^{4}}{l}\right)$

where $V$ is the applied potential, $\sigma$ is the electrical conductivity, $k$ is the thermal conductivity, $T$ is the temperature, $\eta$ the viscosity and $l$ a characteristic length. The electrothermal force for these electrodes is zero at a frequency, $f_{c}$ given by [12]:

$\omega_{c}=2 \pi f_{c} \approx \frac{1}{\tau}\left(2 \frac{\left|\frac{\partial \sigma}{\sigma \partial T}\right|}{\left|\frac{\partial \varepsilon}{\varepsilon \partial T}\right|}\right)^{\frac{1}{2}}$

For water the value of $\frac{1}{\sigma} \frac{\partial \sigma}{\partial T} \approx+2 \%$ per degree and $\frac{1}{\varepsilon} \frac{\partial \varepsilon}{\partial T} \approx-0.4 \%$ per degree [16] so that the frequency $f_{c}$ is of the order of the inverse of the charge relaxation time of the liquid, i.e. $f_{c} \approx \frac{1}{2}(\sigma / \varepsilon)$. Since the magnitude of the electrothermal force can be controlled by varying the frequency and voltage applied to the electrodes, the force can either be eliminated (at a frequency equal to $f$ ) or utilised in micro separation systems.

\section{REFERENCES}

1. H.A. Pohl. Dielectrophoresis. Cambridge University Press, Cambridge UK 1978.

2. R. Pethig. Dielectrophoresis: Using inhomogeneous A.C. electric fields to separate and manipulate cells. Critical Reviews in Biotechnology. 16 (1996) 331-348.

3. T.B. Jones. Electromechanics of Particles (Cambridge, MA: Massachusetts Institute of Technology) 1995.

4. T. Muller, A. Gerardino, Th. Schnelle, S.G. Shirley, F. Bordoni, G. De Gasperis, R. Leoni and G. Fuhr. Trapping of micrometre and sub-micrometre particles by high-frequency electric fields and hydrodynamic forces. Phys. D: Appl. Phys. 29 (1996) 340-349.

5. T. Muller, S. Fiedler, T. Schnelle, K. Ludwig, H. Jung and G. Fuhr, High frequency fields for trapping of viruses. Biotechnology Techniques 10 (1996) 221-226.

6. M. Washizu, S. Suzuki, O. Kurosawa, T. Nishizaka and T. Shinohara. Molecular dielectrophoresis of biopolymers. IEEE Trans. Ind. Appl. 30 (1994) 835-843.

7. N.G. Green, H. Morgan and J.J. Milner. Manipulation and trapping of sub-micron bioparticles using dielectrophoresis. J. Biochem. Biophys. Meth. 35 (1997) 89-102.

8. N.G. Green and H. Morgan. Dielectrophoretic investigations of sub-micrometre latex spheres. J. Phys D: Appl. Phys. 30 (1997) 2626-2633.

9. N.G. Green and H. Morgan. Dielectrophoretic separation of nano-particles. J.Phys.D: Appl. Phys. 30 (1997) L41-44. 
10. H. Morgan and N.G. Green. Dielectrophoretic manipulation of rod-shaped viral particles. J. Electrostatics 42 (1997) 279-293.

11. N.G. Green and H. Morgan. Separation of sub-micron particles using combined dielectrophoretic and electrohydrodynamic forces. J. Phys. D: Appl. Phys. 31 (1998) L25-30.

12. A. Ramos, H. Morgan, N.G. Green and A. Castellanos, A.C. Electrokinetics: A review of forces in microelectrode structures. J. Phys. D: Appl. Phys. (1998) 31 (1998) 23382353.

13. H.P. Schwan. Electrode polarisation impedance and measurements in biological materials. Ann. New York Acad. Sci. 148 (1968) 191-209.

14. A.J. Bard and L.R. Faulkener. Electrochemical Methods, Fundamentals and Applications. John Wiley and Sons. 1980.

15. J. O'M. Bockris and A.K.N. Reddy. Modern Electrochemistry. Plenum Press New York 1973.

16. CRC Handbook of Chemistry and Physics. Edt. David R. Lide $74^{\text {th }}$ edition CRC Press, London 1994.

17. R. Pethig, Y. Huang, X-B. Wang and J.P.H .Burt J. Phys. D: Appl. Phys. 22 (1992) 881-888. 